\title{
Tumor Neuroendocrino del Ciego: Reporte de Caso
}

\section{Paúl Andrade ${ }^{1,2}$}

Este artículo está bajo una licencia de Creative Commons de tipo Reconocimiento - No comercial - Sin obras derivadas 4.0 International

1. Especialista en Cirugía General - Hospital General Docente Ambato.

2. Profesor Auxiliar de Cirugía - Universidad Técnica de Ambato

\section{ORCID ID:}

Paúl Andrade:

https://orcid.org/0000-0003-1651-0075

Recibido: 13 - Aug - 2019.

Aceptado: 26 - Oct - 2019.

Publicado: 01 - Dec - 209.

Correspondencia: Paul Andrade

E-mail: dr.paul_andrade@hotmail.com

Financiamiento: Reporte financiado con fondos propios de los autores.

Conflictos de interés: El autor declara no poseer conflicto de interés.

\section{Resumen}

El carcinoma neuroendocrino (Neuroendocrine carcinoma NEC) del colon es un tumor raro y muy agresivo, generalmente diagnosticado de forma incidental y durante la resolución de complicaciones como perforación u obstrucción.

Se presenta el caso de un paciente masculino de 58 años quien fue intervenido quirúrgicamente por presentar cuadro de peritonitis localizada secundario a un tumor neuroendocrino de ciego perforado.

Se realizó hemicolectomía derecha con resección ganglionar D2 y anastomosis primaria íleo - transversa. El examen histopatológico describió un carcinoma neuroendocrino bien diferenciado de células pequeñas, sin infiltración vascular ni neuronal. Al momento el paciente se encuentra en espera de quimioterapia sin necesidad revisión quirúrgica secundaria.

Palabras clave: Carcinoma neuroendocrino, Colectomía, Peritonitis, Complicación.
Forma de citar este artículo: Andrade P. Tumor Neuroendocrino del Ciego: Reporte de Caso. Rev Med Vozandes. 2019; 30(2): $48-51$

\section{Abstract}

\section{Ciego Neuroendocrine Tumor: Case Report.}

Neuroendocrine carcinoma (NEC) of the colon is a rare and very aggressive tumor, generally diagnosticated in an incidental way and during resolution of complications such as perforation or intestinal obstruction.

The case of a 58-year-old male patient who was surgically intervened for presenting localized peritonitis pedhora scan to a perforated blind neuroendocrine tumor.

Right hemicolectomy whit ganglion D2 resection and ileo - transverse primary anastomosis was performed. Histopathological examination described a well-differentiated neuroendocrine carcinoma of small cells, with no vascular or neuronal infiltration. Now the patient is awaiting chemotherapy without the need for secondary surgical review.

Keywords: :Neuroendocrine carcinoma, Colectomy, Peritonitis, Complication 


\section{Introducción}

Los carcinomas neuroendocrinos (Neuroendocrine carcinoma - NEC) constituyen alrededor de 1 al 2\% de todas las neoplasias. (1) Usualmente se presentan en el aparato digestivo y en el páncreas (representado el $65 \%$ de todos los NEC), pero también, se pueden localizar en otros tejidos del cuerpo como sistema broncopulmonar (25\%), sistema urogenital, cabeza y cuello, mama y piel (10\% restante) ${ }^{(1,2)}$. La localización más frecuente en el tracto digestivo comprende el apéndice, el íleon y el recto. ${ }^{(1,3)}$

El estudio, la nomenclatura y el tratamiento de los NEC han experimentado numerosas modificaciones en la última década. ${ }^{(4)}$ Aunque, algunos autores aún utilizan el término carcinoide (principalmente para NEC de bajo potencial maligno - G1), la tendencia es emplear la nueva clasificación de la OMS según el grado de la lesión, complementada con la estadificación TNM. ${ }^{(4,5)}$

Generalmente, los NEC se diagnostican tardíamente, (5) asociados a complicaciones que demuestran un estadio avanzado. Siendo las complicaciones más frecuentes la perforación (35\%), obstrucción (40\%) o sangrado digestivo bajo; dependiendo de la localización del mismo en el trayecto gastrointestinal.

En nuestro medio no se han encontrado publicaciones sobre tumores neuroendocrinos de ciego, lo que hace importante la revisión de este caso.

\section{Presentación del Caso}

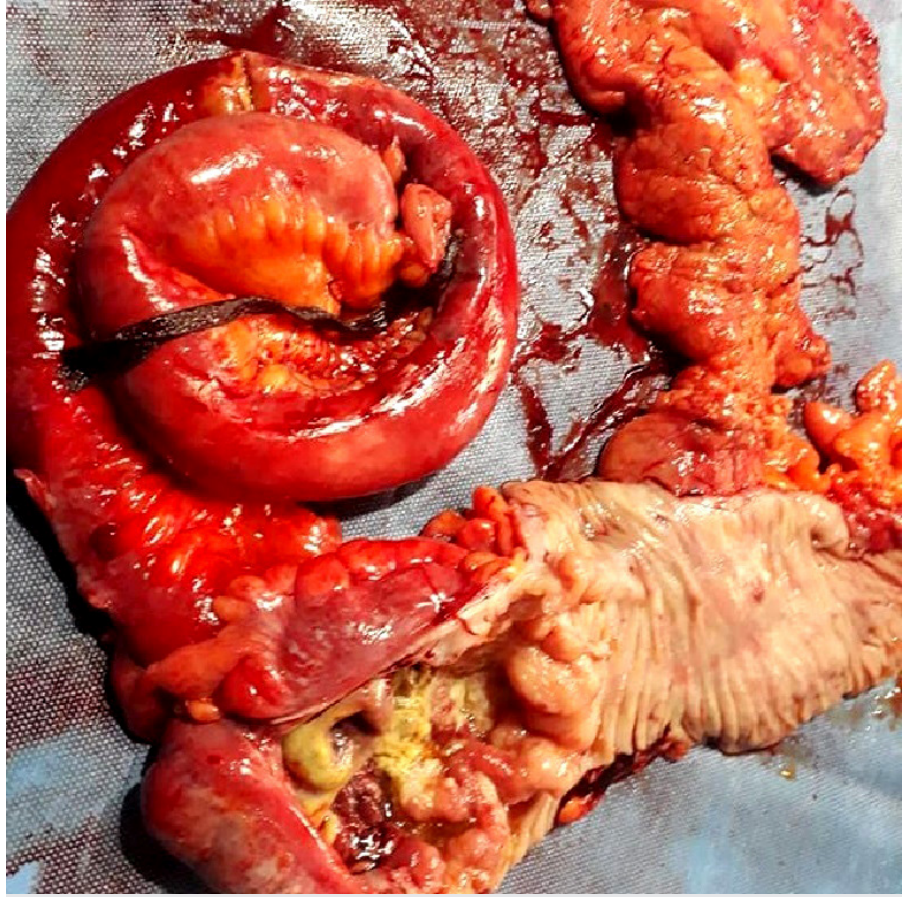

Figura 1. Pieza quirúrgica posterior a la cirugía.

Fotografía: Dr. Paúl Andrade
Se trata de un paciente masculino de 58 años, con antecedentes patológicos clínicos: hipertensión arterial esencial diagnosticada hace 5 años, en tratamiento con Losartan 100mg día, y quirúrgicos: apendicectomía a los 44 años de edad.

Acude al servicio de emergencia del Hospital General Docente Ambato - Ecuador por presentar dolor abdominal localizado en fosa iliaca derecha de 5 días de evolución acompañado de nausea y vómito por varias ocasiones.

Al examen físico el paciente presentó signos vitales dentro de parámetros normales. La palpación abdominal reveló signo de Mussy positivo en hemiabdomen inferior y una masa dura, dolorosa, no móvil en fosa iliaca derecha de $9.0 \times 8.0 \mathrm{~cm}$ aproximadamente.

Por este motivo, se solicitó una ecografía abdominal que reportó: imagen compleja de ecogenicidad irregular de $9.5 \times 7.6 \mathrm{~cm}$, con un volumen aproximado de 360cc y presencia de líquido libre en fondo de saco recto vesical. Debido al antecedente quirúrgico, la principal hipótesis diagnóstica fue: mucocele apendicular.

En virtud de los resultados ultrasonográficos, se consideró la resolución quirúrgica emergente.

La laparotomía exploratoria subsecuente (Figura 1), demostró:

1.Plastrón en fosa iliaca derecha, conformado por epiplón intestino delgado y ciego.

2.Masa dependiente de ciego, necrótica, perforada en cara peritoneal, de $10 \times 8 \mathrm{~cm}$, que infiltra intestino delgado terminal a $5 \mathrm{~cm}$ de válvula ileocecal y peritoneo parietal y tejido graso retroperitoneal.

3.Infiltración de tumor hacia arteria ileocólica y cólica media.

4.Líquido purulento en cuadrante abdominal inferior derecho.

5.Infiltrado tipo empedrado en hígado, compatible con metástasis hepáticas.

Debido a los hallazgos descritos, se realizó una hemicolectomía derecha ampliada, con resección ganglionar D2 y anastomosis primaria íleo transversa latero terminal.

El estudio histopatológico demostró un tumor neuroendocrino de ciego con necrosis de sus paredes, infiltración hacia serosa, no infiltración vascular, no infiltración neuronal, índice mitótico de 2-20/10 HPF, índice Ki67 20\%, ganglios 2/13 positivos. Diagnóstico histopatológico: Tumor neuroendocrino de ciego bien diferenciado. T4, N1, Mx (Figura 2). Inmunohistoquímica con Cromagina A no fue solicitada. 


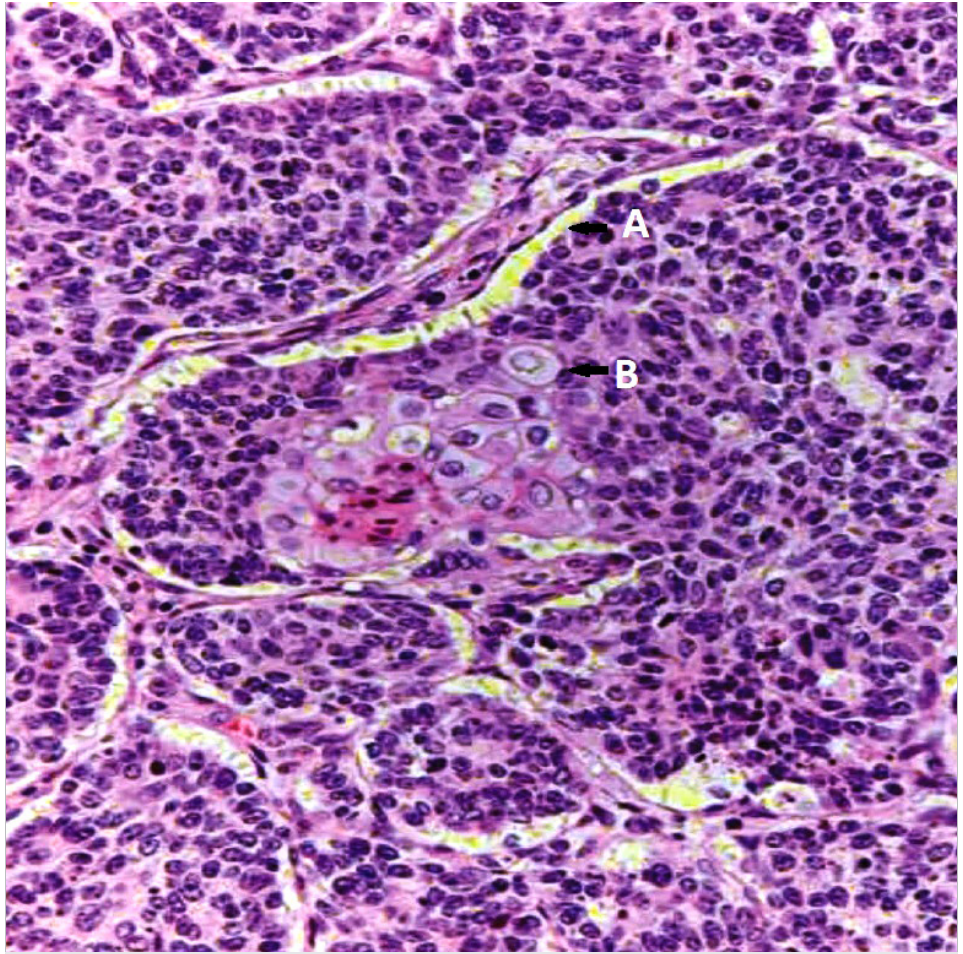

Figura 2. Imagen histopatológica de NEC (Pieza quirúrgica). A: Infiltración a serosa; B: Predominio de celular grandes.

Fotografía: Departamento de Anatomía Patológica - Hospital General Docente Ambato

La evolución postoperatoria fue adecuada, el paciente toleró apropiadamente la ingesta de alimentos y fue dado de alta 3 días después de la intervención quirúrgica.

Al momento en espera de valoración por oncología clínica para estatificación y manejo. Examen tomográfico simple y contrastado de control (abdomen y pelvis) no identificó metástasis ni enfermedad residual.

\section{Discusión}

LOS NEC colorrectales constituyen un grupo heterogéneo de tumores con diferenciación neuroendocrina, representa el 1 al $4 \%$ del total de las neoplasias de esta localización. Predominan entre la sexta y séptima década de la vida y su localización más frecuente es el ciego, el recto y el sigmoides $(1,3,8,9)$.

En el pasado se emplearon diferentes clasificaciones y nomenclaturas de acuerdo con las características histopatológicas, lo que claramente condicionaba el tratamiento de estas lesiones.

Las directrices de práctica clínica de la Organización Mundial de la Salud (OMS), The European Neuroendocrine Tumor Society (ENETS), The American Joint Committee on Cancer (AJCC) y de la North American Neuroendocrine Tumor Society (NANETS) destacan la importancia de combinar el estadio TNM con el grado tumoral según el índice mitótico y el índice Ki-67, los cuales se consideran actualmente como los elementos pronósticos más relevantes ${ }^{(9,10)}$. (Tabla 1)

Estudio multicéntrico Japones (5) con 760 NEC tratados, mostró que los factores predictivos del compromiso ganglionar en el análisis multivariado se asociaron con: el tamaño de la lesión, la profundidad, el índice mitótico, el índice Ki-67, la invasión linfática y vascular. Notablemente, el riesgo de metástasis ganglionar en lesiones menores de $10 \mathrm{~mm}(\mathrm{n}=98)$ fue $9,2 \%$.

Los factores de mal pronóstico histopatológico en NEC: infiltración de la muscular propia del órgano, permeación vascular, número mayor a 2 mitosis por CGA y necrosis tumoral focal (comportamiento más agresivo), determinan su manejo ulterior. ${ }^{(5,7,8)}$ Además, bordes quirúrgicos mayores a $2 \mathrm{~mm}$ son considerados seguros. ${ }^{(5)}$

Actualmente, la tomografía por emisión de positrones (PET-CT) con 68 Ga-Dotatate, se considera el estudio de imagen de elección para el diagnóstico, estadificación y seguimiento de los NEC ${ }^{(6)}$. Esta técnica que permite mostrar la sobreexpresión local de los receptores de somatostatina y está disponible en los últimos años en nuestro medio, pero no es de fácil acceso.

Las recomendaciones para el tratamiento de NEC colónicas son similares a las de Adenocarcinoma de Colon, entre estas debemos considerar: 1) En pacientes sin metástasis a distancia: resección más linfadenectomía local (por laparoscopía - a cielo abierto). 2) En los tumores con metástasis a distancia: resección paliativa más linfadenectomía regional, con quimioterapia previa, para reducir la lesión, y/o posterior, si es que la resección no ha sido completa y

Tabla1 - Clasificación de los Tumores Neuroendócrinos de Colon

\begin{tabular}{|llll|}
\hline Tipo & División Celular (Mitosis) & Índice Ki-67 & Grado de diferenciación celular \\
NEC bien diferenciado - Grado I & Mitosis <2\% por CGA & $<3 \%$ & Bien diferenciado \\
NEC bien diferenciado - Grado II & $2-20$ mitosis por CGA & $3-20 \%$ & Moderada diferenciación \\
NEC bien diferenciado - Grado III & Mitosis > 20\% por CGA & $>20 \%$ & Moderada diferenciación \\
NEC pobremente diferenciados & Mitosis > 20\% por CGA & $>20 \%$ & Escasa diferenciación \\
CGA: campo de gran aumento, NEC: Carcinoma neuroendócrino & \\
\hline
\end{tabular}


3) En los casos de invasión a órganos adyacentes y en complicaciones: hemicolectomía derecha, izquierda o transversa, dependiendo del compromiso del drenaje linfático observado en la cirugía. ${ }^{(8,9)}$

En relación al caso presentado el manejo se realizó con una resección del tumor con criterio oncológico, a pesar de tratarse de una cirugía de emergencia, en el postoperatorio la estadificación del paciente correspondían a un estadio IIIA (T4, NI, MX)), con lo cual se aseguró que no sea necesario una nueva intervención y mejorando el pronóstico de sobrevida del paciente $(7,9)$. Según la bibliografía presentada, si existen elementos de riesgo, debe evaluarse la cirugía radical en pacientes con un riesgo quirúrgico adecuado. En general, los NEC mayores de $2 \mathrm{~cm}$ habitualmente invaden la muscular propia (T2, $5 \%)$ o más allá (T3-T4, 6\%) con un riesgo de alrededor el $60 \%$ de comprometer los ganglios linfáticos locales, por lo que dichos pacientes son candidatos a una resección oncológica formal. El riesgo de metástasis ganglionares en las lesiones entre 1 y $2 \mathrm{~cm}$ es del $30 \% 9$, motivo por el cual requieren una evaluación caso a caso, considerando la edad, las condiciones y comorbilidades del paciente. ${ }^{(8)}$

La TAC es de gran relevancia en el estudio del tumor primario y para la evaluación de enfermedad metastásica. (8) Las lesiones avanzadas (T2-T4, $\mathrm{N}+$ ) tienen indicación de cirugía radical por vía laparoscópica o abierta y una eventual terapia adyuvante según el grado y el estadio de la enfermedad. ${ }^{(6-8)}$

\section{Conclusión}

De acuerdo con lo aprendido de este caso, experiencia y revisión del tema, podemos concluir que el manejo del paciente fue adecuado a pesar de las limitaciones diagnósticas y de la falta de información en relación con la estatificación del paciente previo a la cirugía. Al tratarse de una cirugía de emergencia, el realizar la resección ampliada puede parecer no adecuada, sin embargo, en este caso fue la mejor elección, lo que se confirma con el histopatológico y la TAC posterior donde se confirmamos que la cirugía realizada tiene un margen adecuado de resección y otorga al paciente de la posibilidad de un manejo adecuado con relación a la quimioterapia adyuvante sin necesidad de cirugías complementarias.

\section{Referencias}

1. Shah R, Nalamati SPM. Neuroendocrine tumors of the colon and rectum. Seminars in Colon and Rectal Surgery. 2019; 26 (2): 60-63. doi:10.1053/j.scrs.2015.01.002.

2. Shen $C$, Yin $Y$, Chen $H$, Tang $S$, Yin S, Zhou Z, et al. Neuroendocrine tumors of colon and rectum: validation of clinical and prognostic values of the World Health Organization 2010 values of the World Health Organization 2010
grading classifications and European Neuroendocrine Tumor Society staging systems. Oncotarget. 2017; 8 (13): 22123-34

3. Jiang $M$, Tan $Y, L i X$, Fu J, Hu H, Ye X, et al. Clinicopathological Features and Prognostic Factors of Colorectal Neuroendocrine Neoplasms. Gastroenterol Res Pract. 2017;2017:4206172. doi: 10.1155/2017/4206172.
4. Bosolino A, Ratto R. Tumores neuroendocrinos de colon y recto. Acta Gastroenterol Latinoam 2018;48(4):327-337

5. Kojima M, Ikeda K, Saito N, Sakuyama N, Koushi K, Kawano S, et al. Neuroendocrine Tumors of the Large Intestine: Clinicopathological Feathe Large Intestine: Clinicopathological Fea-
tures and Predictive Factors of Lymph Node tures and Predictive Factors of Lymph Node
Metastasis. Front Oncol. $2016 \mathrm{Jul}$ 18;6:173. doi: 10.3389/fonc.2016.00173.

6. Ramage JK, De Herder WW, Delle Fave G, Ferolla $\mathrm{P}$, Ferone $\mathrm{D}$, Ito $\mathrm{T}$, et al. ENETS Consensus Guidelines Update for Colorectal Neuroendocrine Neoplasms. Neuroendocrinology. 2016;103(2):139-43. doi: 10.1159/000443166.
. Bannura G, Barrera A, Melo C, Illanes F, Gallardo C. Tumores neuroendocrinos primarios de colon y recto. Rev Chil Cir 2018;70(1):53-58

8. Moradi F, Jamali M, Barkhodari A, Schneider B, Chin F, Quon A, et al. Spectrum of 68 Ga-DOTA TATE uptake in patients with neuroendocrine tumors. Clin Nuclear Med. 2016;41:e281-7.

9. Mantzoros I, Savvala NA, loannidis O, Parpoudi S, Loutzidou L, Kyriakidou D, et al. Midgut neuroendocrine tumor presenting with acute intestinal ischemia. World J Gastroenterol. 2017 Dec 7;23(45):8090-8096. doi: 10.3748/wjg.v23. i45.8090. 